\title{
Supplement of differential equations of fraction order for forecasting of financial markets
}

\author{
Sergey Erokhin ${ }^{1, *}$ and Olga Roshka ${ }^{2}$ \\ ${ }^{1}$ Moscow State University of Civil Engineering, Yaroslavskoe shosse, 26, Moscow, 129337, Russia
}

\begin{abstract}
In this paper, the analysis of capital markets takes place using the advection-diffusion equation. It should be noted that the methods used in modern theoretical physics have long been used in the analysis of capital markets. In particular, the Fokker-Planck equation has long been used in finding the probability density function of the return on equity. Throughout the study, a number of authors have considered the supplement of the Fokker-Planck equation in the forecasting of equity markets, as a differential equation of second order. In this paper, the first time capital markets analysis is performed using the fractional diffusion equation. The rationale is determined solely by the application nature, which consists in generation of trading strategy in equity markets with the supplement of differential equation of fractional order. As the subject for studies, the differential operator of fractional order in partial derivatives was chosen - the FokkerPlanck equation. The general solutions of equation are the basis for the forecast on the exchange rate of equities included in the Dow Jones Index Average (DJIA).
\end{abstract}

\section{Introduction}

Differential equations have found wide supplement in modern financial analysis [1-5]. The topic of this paper is the generation of a probability density function, as a solution to a fractional differential equation (fractional diffusion equation or Fokker-Planck fractional differential equation).

The Fokker-Planck equation is necessary as a differential operator specifying the time evolution of the distribution density of the return on equities.

\section{The fractional differential Fokker-Planck equation}

The object of work under consideration is the price processes in the financial markets. In its standard form, this operator will look like a partial differential equation:

$$
\frac{\partial^{\alpha}}{\partial x^{\alpha}}\{\sigma(x, t) \cdot U(x, t)\}-\frac{\partial}{\partial x}\{\mu(x, t) \cdot U(x, t)\}=\frac{\partial}{\partial t}\{U(x, t)\},
$$

\footnotetext{
* Corresponding author: ErokhinSV@mgsu.ru
} 
where

$\alpha$ - fractional derivative order;

$x$ - return on equities;

$U(x, t)$ - the density function of the distribution of return on equities;

$\mu(x, t)$ - the mathematical expectation of the random drift of return on equities;

$\sigma(x, t)$ - variance around a given drift,

$\frac{\partial^{\alpha}}{\partial x^{\alpha}}$ - the operator of fractional differentiation in the sense of Riemann-Liouville, defined as follows. Let $f(x) \in L_{1}(0,1)$. Then function

$$
\frac{d^{-\alpha}}{d x^{-\alpha}} f(x) \equiv \frac{1}{G(\alpha)} \int_{0}^{\mathrm{x}} f(t) d t \in L_{1}(0,1)
$$

is called a fractional integral of order $\alpha>0$ with start at the point $x=0$, and the function

$$
\frac{d^{-\alpha}}{d(1-x)^{-\alpha}} f(x) \equiv \frac{1}{G(\alpha)} \int_{x}^{1}(t-x)^{\alpha-1} f(t) d t \in L_{1}(0,1)
$$

is called a fractional integral of order $\alpha>0$ with end at the point $x=1$. Here, $G(\alpha)$ is the Euler gamma-function. As we know [6,7], the function $g(x) \in L_{1}(0,1)$ of order $\alpha>0$ with start at the point $x=0$, if

$$
f(x)=\frac{d^{-\alpha}}{d x^{-\alpha}} g(x)
$$

Indicate then

$$
g(x)=\frac{d^{\alpha}}{d x^{\alpha}} f(x)
$$

hereinafter under the symbol

$$
\frac{d^{\alpha}}{d x^{\alpha}}
$$

we shall mean a fractional operator of fractional integration for $\alpha<0$ and fractional differentiation for $\alpha>0$. A fractional derivative

$$
\frac{d^{\alpha}}{d(1-x)^{\alpha}}
$$

of order $\alpha>0$ of the function $f(x) \in L_{1}(0,1)$ with end at the point $x=1$ is defined in the same way.

We use the standard financial analysis of the attitude, in which $U(x, t)$ decomposes into $(X(x)$ and $T(t))$ - the method of separation of variables. Then equation (1) can be rewritten as:

$$
\frac{\partial^{\alpha}}{\partial x^{\alpha}} \cdot\{\sigma(x) \cdot X(x) \cdot T(t)\}-\frac{\partial}{\partial x}\{\mu(x) \cdot X(x) \cdot T(t)\}=\frac{\partial}{\partial t}\{X(x) \cdot T(t)\}
$$

Equation (2) is a function of instantaneous drift $\mu(x)$ and diffusion $\sigma(x)$, depending only on the financial variable $X$.

After elementary transformations, the fractional Fokker-Planck equation takes the following form: 


$$
\frac{d^{\alpha}}{d x^{\alpha}} \sigma(x) \frac{d}{d x} \mu(x)-\frac{\frac{d}{d x}\{X(x) \mu(x)\}}{X(x)}+2 \frac{\frac{d}{d x} X(x) \frac{d}{d x} \sigma(x)}{X(x)}+\frac{\frac{d}{d x}\{X(x) \sigma(x)\}}{X(x)}=\frac{\frac{d}{d t} T(t)}{T(t)}=-\lambda
$$

Equation (3) splits into a system of two differential equations. The equation for $T(t)$ is an ordinary differential equation of the first order, which solution is written in explicit form. And the equation for the determination of $X(x)$ is a fractional differential equation, which is one of the fundamental equations of the mathematical model of point particle random walk along a self-similar fractal set $\Omega$ embedded in $R^{u}, \mathrm{u} \geq 2$.

Thus, the solution of equation (1), as in [2], can be written in the form

$$
\begin{gathered}
U(x, t)=\sum_{n=1}^{\infty} \varphi_{n} e^{\lambda_{n} D t} x^{\alpha-1} E_{\alpha \alpha}\left(\lambda_{n} x^{\alpha}\right) \\
\text { where } \\
E_{\alpha \alpha}\left(\lambda_{n} x^{\alpha}\right)=\sum_{k=0}^{\infty} \frac{\left(\lambda_{n} x^{\alpha}\right)^{k}}{\Gamma(\alpha+\alpha k)}
\end{gathered}
$$

is a well-known function of the Mittag-Leffler type, and $D=1$.

\section{Supplement of Fokker-Planck equation for forecasting of financial markets}

The Fokker-Planck fractional differential equation was adapted to the needs of financial mathematics [8-10]. Based on the foregoing, a simple trade rule based on the solution of the following Fokker-Planck equation (1) is constructed. To analyze the function $U(x, t)$, we use the Fourier method [11].

Represent $U(x, t)$ as $X(x)$ and $T(t)$ and obtain equation:

$$
\frac{\partial^{\alpha}}{\partial \mathrm{x}^{\alpha}}[\sigma(x) X(x) T(t)]-\frac{\partial}{\partial x}\left[\sigma(x) X(x) \frac{\mu(x)}{\sigma(x)} T(t)\right]=\frac{\partial}{\partial t}\left[\sigma(x) X(x) \frac{1}{\sigma(x)} T(t)\right]
$$

Indicate $\tilde{X}(x)=\sigma(x) X(x)$. From last relation:

$$
\left\{\begin{array}{l}
\frac{d^{\alpha}}{d x} \tilde{X}(x)-\frac{d}{d x}\left[\tilde{X}(x) \frac{\mu(x)}{\sigma(x)}\right]=-\lambda \tilde{X}(x) \sigma(x) \\
\frac{d}{d t}[T(x)]=-\lambda T(t)
\end{array}\right.
$$

Further, the solution assumes parameterization of the instantaneous drift function $\mu(x)$ and diffusion function $\sigma(x)$.

The following specifications are relevant in financial markets $[12,13]$ :

$$
\begin{gathered}
\mu(x)=m x \\
\sigma(x)=S^{2} x^{2}
\end{gathered}
$$

where " $\mathrm{m}$ " is the shift in average financial coordinate (revenue) on the sliding period; 
$S$ - the mean-square deviation of revenue, measured on the same sliding vector. Then $U(x, t)$ can also be represented in the form [14]

$$
\begin{array}{r}
U(x, t)=\sum_{k=0}^{\infty} \varphi_{n} E_{\alpha}\left(\lambda_{n} t^{\alpha}\right) x^{\alpha-1} E_{\alpha \alpha}\left(\lambda_{n} x^{\alpha}\right) \\
E_{\alpha}(z)=\sum_{k=0}^{\infty} \frac{z^{k}}{G(1+\alpha k)}, E_{\alpha \alpha}(z)=\sum_{k=0}^{\infty} \frac{z^{k}}{G(\alpha+\alpha k)},
\end{array}
$$

where

$\lambda_{n}-$ n-th zero function $E_{\alpha \alpha}(z)$.

For $\alpha=1.5$, the first zeros functions $E_{\alpha \alpha}(z)$ are shown in Table 1 .

Table 1. Zero functions $E_{\alpha \alpha}(z)$

\begin{tabular}{|c|c|}
\hline $\mathbf{n}$ & n-th zero function $\boldsymbol{E}_{\boldsymbol{\alpha} \boldsymbol{\alpha}}(\mathbf{z})$ \\
\hline 1 & 5.5471 \\
\hline 2 & 17.642 \\
\hline 3 & 29.698 \\
\hline 4 & 43.878 \\
\hline 5 & 51.148 \\
\hline 6 & $65.701-3.2637 \mathrm{i}$ \\
& $65.701+3.2637 \mathrm{i}$ \\
\hline 7 & $77.436-2.7281 \mathrm{i}$ \\
& $77.436+2.7281 \mathrm{i}$ \\
\hline 8 & 81.758 \\
\hline
\end{tabular}

Eigenfunctions, $\beta=\alpha$

$$
\chi_{n}=x^{\beta-1} E_{\beta \beta}\left(\lambda_{n} x^{\beta}\right),
$$

where

$$
E_{\beta \beta}\left(\lambda_{n} x^{\beta}\right)=\sum_{k=0}^{\infty} \frac{\left(\lambda_{n} x^{\beta}\right)^{k}}{G(\beta+\beta k)}
$$

Biorthogonal functions

$$
\chi_{n}^{*}(x)=(1-x)^{\beta} E_{\beta \beta}\left(\lambda_{n}(1-x)^{\beta}\right)
$$

The Fourier coefficients are calculated by the formula [15]

$$
\varphi_{n}=\int_{0}^{1} \chi_{n}^{*}(x) f(x) d x
$$

where

$$
f(x)=\left\{\begin{array}{l}
2 x, \quad 0 \leq x \leq 1 / 2 \\
(4-2 x) / 3, \quad 0.5 \leq x<2
\end{array}\right.
$$

and shown in table 2 . 
Table 2. The Fourier coefficients for the expansion according to formula (3)

\begin{tabular}{|c|c|}
\hline $\mathbf{n}$ & $\boldsymbol{\varphi}_{\boldsymbol{n}}$ \\
\hline 1 & 0.71741 \\
\hline 2 & 8.848 \\
\hline 3 & 66.819 \\
\hline 4 & 580.78 \\
\hline 5 & 1609.2 \\
\hline 6 & $9550.2-3905 \mathrm{i}$ \\
& $9550.2+3905 \mathrm{i}$ \\
\hline 7 & $37019-11100 \mathrm{i}$ \\
& $37019+11100 \mathrm{i}$ \\
\hline 8 & 60562 \\
\hline
\end{tabular}

Graph of solution $u(x, t)$ will have the following form (fig. 1):

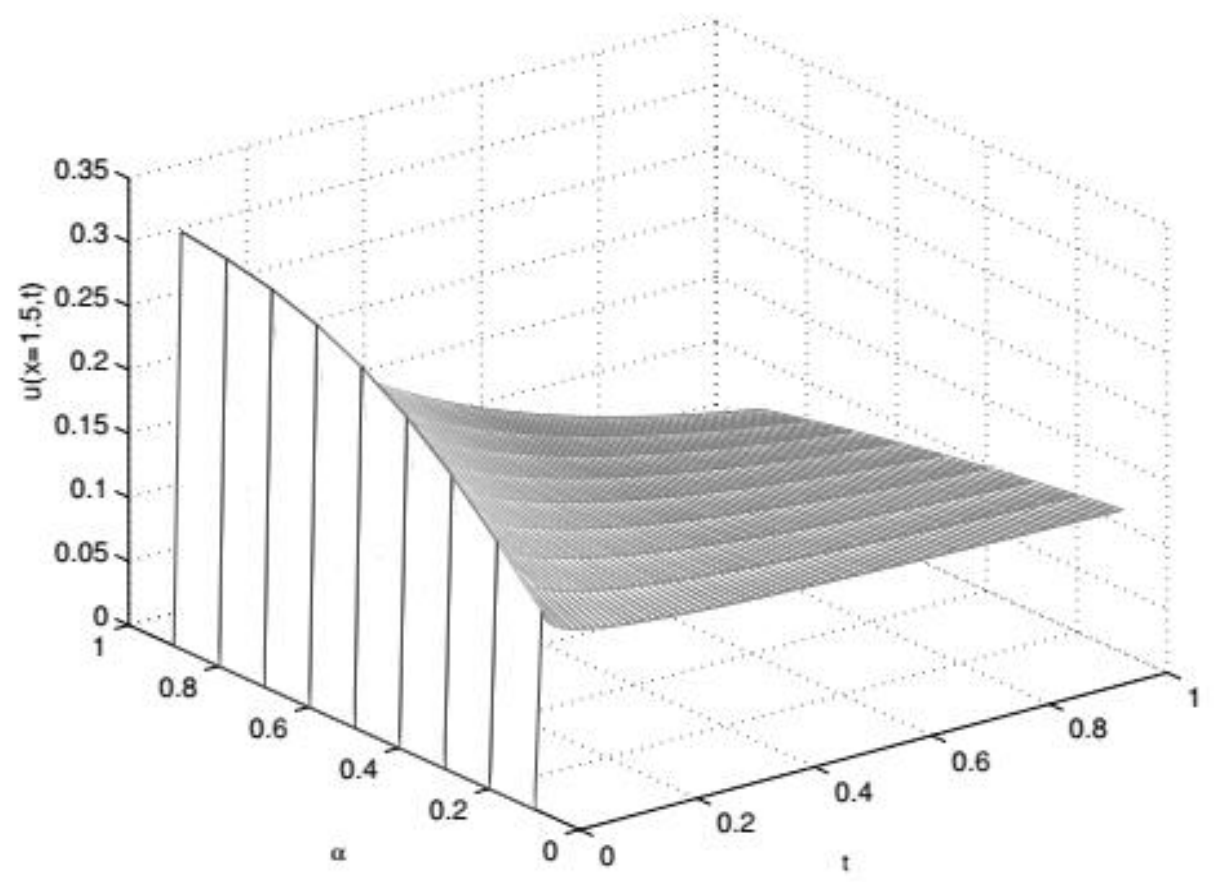

Fig. 1. The graph of the solution of the function $u(x, t)$

\section{Conclusions}

The paper studies the standard Fokker-Planck equation; for the first time the fractional order equation adapted to the needs of financial mathematics was applied to the study of financial markets. The parameters according to the equation are selected, the graph of the solution is shown clearly demonstrating the derivation of the optimal equation for forecasting the equity market.

The equation considered for $\alpha=2$ was first used in static physics to study the time evolution of the probability density function of the velocity of an elementary particle. 


\section{References}

1. J. Korbel, Yu. Luchko. Fr. Calc. and Appl. An. 19, 6 (2016)

2. T. Aleroev, H. Aleroeva, J. Huang, M. Tamm, Y. Tang, Y. Zhao. Advances in Fractional Differential Equations (IV): Time-fractional PDEs" in Computers \& Mathematicswith Applications, (2016) http://dx.doi.org/10.1016/j.camwa.2016.06.038

3. L. Calvet and A. Fisher. Multifractal Volatility: Theory, Forecasting, and Pricing, Academic Press Advanced Finance (Elsevier Science, 2008).

4. P. Carr and L. Wu. Journal of Finance 58, 753 (2003).

5. H. Kleinert. Physica A: Statistical Mechanics and its Applications 312, 217 (2002)

6. T. Aleroev. Sib. elektron. matem. izv., 10, 41-55 (2013)

7. I. Podlubny. Fractional Differential Equations, Volume 198: An Introduction to Fractional Derivatives, Fractional Differential Equations, to Methods of Their ... (Mathematics in Science and Engineering), (Academic Press, 1998).

8. K.-I. Sato. L'evy Processes and Infinitely Divisible Distributions, Cambridge Studies in Advanced Mathematics (Cambridge University Press, 1999).

9. G. Samoradnitsky and S. Taqqu. Stable Non-Gaussian Random Processes: Stochastic Models with Infinite Variance, Stochastic Modeling Series (Taylor \& Francis, 1994).

10. P. Tankov. Financial Modelling with Jump Processes, Chapman \& Hall/CRC Financial Mathematics Series (Taylor \& Francis, 2003).

11. G. Pagnini. Fractional Calculus and Applied Analysis 16, 436 (2013).

12. C. Necula. Option Pricing in a Fractional Brownian Motion Environment, Advances in Economic and Financial Research - DOFIN Working Paper Series 2 (Bucharest University of Economics, Center for Advanced Research in Finance and Banking CARFIB, 2008).

13. G. Jumarie. Computers \& Mathematics with Applications 59, 1142 (2010).

14. T. Abdeljawad. Comput. Math. Appl. 62, 1602 (2011).

15. H. Kleinert and V. Zatloukal. Phys. Rev. E 88, 052106 (2013), (http://klnrt.de/407). 